BUHEP-95-tba

hep-th/9507082

\title{
Binary Cosmic Strings
}

\author{
Ryan Rohm and Indranil Dasgupta \\ Physics Department \\ Boston University \\ Boston, MA 02215
}

\begin{abstract}
The properties of cosmic strings have been investigated in detail for their implications in early-universe cosmology. Although many variations of the basic structure have been discovered, with implications for both the microscopic and macroscopic properties of cosmic strings, the cylindrical symmetry of the short-distance structure of the string is generally unaffected. In this paper we describe some mechanisms leading to an asymmetric structure of the string core, giving the defects a quasi-two-dimensional character. We also begin to investigate the consequences of this internal structure for the microscopic and macroscopic physics.
\end{abstract}

July, 1995

e-mail: rohm@ryan.bu.edu,dgupta@budoe.bu.edu 


\section{Introduction}

Since the paper of Nielsen and Olesen [1] describing the vortex solutions of the Abelian Higgs model, many variations of this structure have been studied, giving rise to diverse phenomena. Superconducting cosmic strings [2] are a dramatic example of this. Cosmic strings arise as classical solutions of many Grand Unified Theories (GUTs) and other models of fundamental interactions. They may play an interesting role in cosmology since they are formed in the early universe, when the order parameter of spontaneous symmetry breaking is uncorrelated at large distances; later, they may perturb the space-time metric [3] 国 and contribute to the density fluctuations influencing galaxy formation. In most previously studied variations of the basic Nielsen-Olesen vortex solution the cylindrical symmetry is preserved. We explore another variation in the basic structure of cosmic strings arising in some non-minimal extensions of the standard model, in which additional fields appear in the classical equations and the resulting configurations break the azimuthal symmetry. (These additional fields may arise, for instance, in an extended Higgs sector, or may be part of the matter sector of the theory.) We will discuss two classes of models: one in which pairs of string are confined due to a spontaneously-broken discrete gauge symmetry, and another class in which independently stable cosmic strings form bound pairs of nearly parallel strings because of short-range attractive forces.

Although there are several mechanisms for obtaining strings with an asymmetric core, the macroscopic classical motion of the strings can be described by a geometrical effective action, and only the relative size of the couplings depends on the details of the shortdistance structure. For this reason, we discuss here the conditions for obtaining binary strings, and report on the effect of this additional structure on the macroscopic behavior of the cosmic strings in a separate paper[0].

A simple physical argument will serve to illustrate the necessary condition for a bound state to occur. Recall [6] that in the Nielsen and Olesen solution, two vortices (that is, cross-sections of a cosmic string) will attract or repel each other depending on the relative strength of the electric charge and the scalar self-coupling. For $\beta \equiv\left(\frac{m_{S}}{m_{V}}\right)^{2}<1$, two $n=1$ vortices have a short range attraction, so that the ground state in the two-vortex sector is the $n=2$ vortex. For $\beta>1$, the magnetic force dominates, leading to a repulsive short range force. (The critical [6] case $\beta=1$ has additional symmetries, but it will not concern us here.) In the case of GUT cosmic strings, we will show that for some ranges of couplings the force between vortices is attractive at long distances but at short distances 
the force becomes repulsive. This can easily happen, for instance, when the longer-range force is confining, that is, when the discrete symmetry (the holonomy of a single string) is spontaneously broken. This leads to a long-range force confining pairs of vortices; if the short-range interaction is repulsive $(\beta>1)$, they do not coalesce into a single rotationally symmetric vortex. Such a structure can also arise when the balance between short-range repulsive forces and short-range attractive forces between two vortices has a local minimum, leading to a finite binding energy per unit length. In either case the classical ground state consists of a pair of finitely-separated vortices, or in the $(3+1)$-dimensional case, a bound pair of cosmic strings. We will then discuss examples of both realizations of binary cosmic strings, beginning with the confining case.

\section{Confined binary strings}

We will present two models in which binary strings arise because of long-range confining forces. In both cases we have 'unary' cosmic strings formed at one scale, which are charged under a discrete gauge symmetry. At a lower scale, another scalar field breaks this discrete symmetry, and the original strings become boundaries of domain walls. These domain walls serve to bind together pairs of the original strings into binary strings, which are then in a sense quasi-one-dimensional domain walls, whose width and thickness are comparable in size. Later in the paper we will describe some GUT realizations of such models, such as the domain-wall-connected strings previously discussed in the literature [7].

In the first model, we consider a spontaneously broken $U(1)$ gauge theory with two charged scalars $\phi$ and $\chi$; the field $\phi$ has twice the charge of $\chi$. The Lagrangian is:

$$
L=-\frac{1}{4} F_{\mu \nu} F^{\mu \nu}+\left|D_{1} \phi\right|^{2}+\left|D_{2} \chi\right|^{2}-V .
$$

where $D_{1 \mu} \phi=\left(\partial_{\mu}-i e A_{\mu}\right) \phi, D_{2 \mu} \chi=\left(\partial_{\mu}-\frac{1}{2} i e A_{\mu}\right) \chi$ and

$$
V=\frac{\lambda_{1}}{8}\left(|\phi|^{2}-v_{1}^{2}\right)^{2}+\frac{\lambda_{2}}{8}\left(|\chi|^{2}-v_{2}^{2}\right)^{2}+\kappa\left(|\phi|^{2}-v_{1}^{2}\right)\left(|\chi|^{2}-v_{2}^{2}\right) .
$$

We also define

$$
\gamma=\frac{v_{2}^{2}}{v_{1}^{2}}
$$

We shall show that for a range of values of $\gamma$ and the coupling constants $\lambda_{1}$ and $\kappa$, and for all values of $\lambda_{2}$, the lowest-mass stable configurations are binary strings. The finiteness 
of the mass per unit length of local strings (in contrast to that of global strings) derives from the proper long-range behavior of the gauge field; to ensure this, the winding number $\nu_{\phi}$ of the phase of $\phi$ must equal twice the winding number of the phase of : $\nu_{\phi}=2 \nu_{\chi}$. The smallest finite-mass solution then has $\nu_{\phi}=2$. In the absence of $\chi$ there would be stable $\nu_{\phi}=1$ strings, but in this model separating the $\nu_{\phi}=2$ string into two of the original strings with $\nu_{\phi}=1$ creates a domain wall, and for large separations requires a linearly-increasing energy. We then need to determine the form of the $\nu_{\phi}=2$ string. We will demonstrate that for the range of parameters mentioned above, the cylindrically symmetric configuration is an unstable solution of the classical field equations; since we have argued that the string cannot separate, this indicates that the binary string is the lowest-energy classical solution.

We will first demonstrate the instability of $\nu_{\phi}>1$ strings (and hence existence of binary strings) when we set the coupling $\kappa=0$, and later demonstrate that this restriction is inessential. To start, consider the $(\phi, A)$ system if we totally ignore (i.e., decouple) the field $\chi$; then $\beta=\left(\frac{m_{S}}{m_{V}}\right)^{2}=\left(\frac{\lambda_{1}}{4 e^{2}}\right)$, and for convenience we define $\alpha=\beta-1$. In that case the proof proceeds along the lines of [6], where it was shown that cylindrically symmetric $n=2$ strings in the theory with $\alpha>0$ (and only one Higgs field, i.e. here we ignore $\chi$ ), are unstable against specific perturbations which correspond to the splitting of the string. The instability occurs because for $\alpha>0$ there are asymmetric perturbations around the cylindrically symmetric solution that lower the energy; for $\alpha=0$ these asymmetric perturbations of the cylindrically symmetric classical solutions are zero modes of the Hamiltonian.

If we make the same perturbations and include the effects of the lighter Higgs field $\chi$, (so $\lambda_{2}$ and $\gamma$ are nonzero), there will be additional positive contributions to the energy, and the line of instability does not start at $\alpha=0\left(\lambda_{1}=4 e^{2}\right)$. This is because while energy from the $(\phi, A)$ sector does not change, the perturbations in A will affect derivative couplings between $\mathrm{A}$ and $\chi$, giving a positive contribution to the energy. Nevertheless, for any $\alpha>0$ the strength of the instability is finite for $\lambda_{2}=\gamma=0$, and so by continuity there will be some range of $\lambda_{2}$ and $\gamma$ near zero for which the symmetric vortex is unstable.

We begin by describing the perturbations. The cylindrically symmetric $\nu_{\phi}=n$ solution will have two independent functions which we call $\Phi$ and $v[6]$ :

$$
\phi(r, \theta)=\Phi(r) \exp (\operatorname{in} \theta)
$$




$$
A_{\theta}=\frac{n}{r} v(r)
$$

where $r$ and $\theta$ are polar coordinates on the 2-plane containing the vortex; we will consider $n=2$. As usual we have $A_{0}=A_{3}=A_{r}=0$. We parametrize a perturbation about this solution as:

$$
\begin{gathered}
\phi(r, \theta)=\Phi(r) \exp (2 i \theta)+\eta \bar{\phi}(r, \theta) \\
A_{\theta}=\frac{2}{r}(v(r)+\eta \bar{v}(r, \theta)) . \\
A_{r}=\eta \bar{w}(r, \theta) .
\end{gathered}
$$

where $\eta<<1$ is a small parameter.

In particular, when $\alpha=0$, perturbations of the form

$$
\begin{gathered}
\bar{\phi}(r, \theta)=\omega \Phi(r) \\
A_{i}=A_{i}^{(0)}+\eta \epsilon_{i j} \partial_{j} \omega .
\end{gathered}
$$

where $i, j=1,2$ and $A_{i}^{(0)}$ is the cylindrically symmetric solution of the equation of motion, produce a zero mode if $\omega$ satisfies $\Delta \omega=2 \Phi^{2} \omega$. These zero modes of the energy may be resolved into eigenfunctions of the azimuthal angular momentum operator, so we may label the solutions as $\omega=\omega_{m}(r) \cos (m \theta)$. For $m=1$ the perturbation is nothing but a translation of the vortex in its own plane, while modes with $m>1$ correspond to distortions of the string core. For finiteness of energy one must also require that $m \leq \nu_{\phi}$ where $\nu_{\phi}$ is the winding number of $\phi$.

For $\alpha>0$, one can generalize these perturbations so that they reduce to the above form when $\alpha=0$. The translational zero mode exists in both cases; however, modes with $m>1$ now decrease the energy, signalling the instability of the symmetric solution.

We now consider this analysis for the combined $(\phi, \chi)$ system. Our boundary conditions require the winding numbers of $\phi$ and $\chi$ to be $\nu_{\phi}=2$ and $\nu_{\chi}=1$ respectively. The $(\phi, A)$ sector is identical to the system in [6] with $\nu_{\phi}=2$. It is sufficient to consider the $m=2$ perturbation in the $(\phi, A)$ sector without disturbing the configuration of the scalar field $\chi$, since this only underestimates the extent of instability. Let us first expand the change in the vortex energy due to this perturbation in a power series in $\alpha$ and $\gamma$ for fixed $\lambda_{2}$, keeping $\kappa=0$ for now. We separate the change in energy into $\gamma$-dependent and $\gamma$-independent parts:

$$
\delta E=\delta E_{1}+\delta E_{2}
$$


where the first term on the R.H.S is $\gamma$-independent. In fact, $\delta E_{1}$ is equal to $\delta E$ for the system in [6]. $\delta E_{1}$ vanishes for $\alpha=0$, so we can expand it in a power series in $\alpha$;

$$
\delta E_{1}=\alpha^{c_{1}}\left(K_{0}+K_{1} \alpha+\ldots\right)
$$

where the coefficients are all independent of the parameters of the $\chi$ sector. $\delta E_{2}$ is the $\gamma$-dependent part; this part is regular (at zero) in both $\gamma$ and $\alpha$ (regularity in $\gamma$ follows from the fact that $\delta E_{2}$ is zero for $\left.\gamma=0\right)$. Therefore it can be expanded as

$$
\delta E_{2}=\gamma^{c_{2}}\left(L_{0}(\alpha)+L_{1}(\alpha) \gamma+\ldots\right)
$$

All the $\alpha$-dependent coefficients of the expansion are regular in $\alpha$.

We have already seen that $\delta E_{1}<0$ for $\alpha>0$ (and hence $K_{0}<0$ ), while earlier we argued that for $\gamma>0, \delta E_{2}>0$. There is then a curve in the $(\gamma, \alpha)$ plane, passing through $\gamma=\alpha=0$ and extending into the positive quadrant, along which there is a zero mode, i.e., $\delta E_{1}+\delta E_{2}=0$. This curve separates the positive quadrant in the $\gamma$, $\alpha$ plane into two parts with opposite signs for $\delta E$, and represents the line of marginal stability for the cylindrically symmetric solution. Since $\delta E$ is negative in the part containing the axis $\gamma=0$, for every $\alpha>0$ there is a range of values of $\gamma$ for which any cylindrically symmetric solution to the equations of motion is unstable. However, it is clear that because of the field $\chi$ the $\nu_{\phi}=2$ string can not split completely into two well-separated strings, since isolated $\nu_{\phi}=1$ strings do not have finite energy per unit length. The ground state in this region of parameter space then must be a localized but asymmetric cosmic string.

This proves the existence of binary strings in this model when $\kappa=0$. The restriction to $\kappa=0$ is not necessary, since just as we found a range of binary-string solutions in the $\alpha-\gamma$ plane, we can continue this region of solutions into the third parameter-dimension, corresponding to the parameter $\kappa$. Since the contribution of this term is nonsingular, for small values of $\kappa$ the cylindrical solution is still unstable. There is then no finetuning necessary to obtain this class of solutions, provided only that the desired pattern of symmetry breaking takes place. In particular, if we assume that the second symmetry breaking takes place at a lower energy scale, one does not expect the contributions from the lighter scalar to affect the question of whether the $\nu_{\phi}=2$ strings are stable, and so we would expect the binary string to be favored over a wide range of parameters, so long as $\alpha>0$. 
(Note that we could have added to the Lagrangian a term $\left(\phi^{*} \chi^{2}+\right.$ c.c. $)$ which removes the global symmetry allowing independent rotations of $\phi$ and $\chi$; it can be similarly dealt with, and will be discussed further at the end of this section.)

Our second example of binary strings confined by a long-range force is provided by a broken $U(1) \times \tilde{U}(1)$ theory. This time we consider three charged scalar fields $\phi, \chi$ and $\tau$ with charges $(1,0),(0,1)$ and $\left(\frac{1}{2}, \frac{1}{2}\right)$ respectively.

The lagrangian is

$$
L=\frac{1}{2}|D \phi|^{2}-\frac{1}{4} F_{\mu \nu} F^{\mu \nu}+\frac{1}{2}|D \chi|^{2}-\frac{1}{4} G_{\mu \nu} G^{\mu \nu}+\frac{1}{2}|D \tau|^{2}-V .
$$

$\mathrm{F}$ and $\mathrm{G}$ are the kinetic terms for the two gauge fields $A_{\mu}$ and $B_{\mu}$. We also have,

$$
\begin{gathered}
V=\lambda_{1}\left(|\phi|^{2}-v_{1}^{2}\right)^{2}+\lambda_{2}\left(|\chi|^{2}-v_{2}^{2}\right)^{2}+\lambda_{3}\left(|\tau|^{2}-v_{3}^{2}\right)^{2}+\alpha\left(|\phi|^{2}-v_{1}^{2}\right)\left(|\chi|^{2}-v_{2}^{2}\right)+ \\
\kappa_{1}\left(|\phi|^{2}-v_{1}^{2}\right)\left(|\tau|^{2}-v_{3}^{2}\right)+\kappa_{2}\left(|\chi|^{2}-v_{2}^{2}\right)\left(|\tau|^{2}-v_{3}^{2}\right)
\end{gathered}
$$

We parametrize the ratio of the vev's by

$$
\zeta^{2}=\frac{v_{2}^{2}}{v_{1}^{2}} ; \quad \gamma^{2}=\frac{v_{3}^{2}}{v_{1}^{2}}
$$

Once again we first restrict the parameter space by putting $\kappa_{1}=\kappa_{2}=0$. The vev's of $\phi, \chi$ and $\tau$ are respectively $v_{1}, \zeta v_{1}$ and $\gamma v_{1}$. We expect to find stable solutions only for boundary conditions for which the phase of each scalar field has an integer winding number at large distances; a minimal choice gives the winding numbers 1, -1 and 0 , respectively. With this choice of couplings and boundary conditions, $\tau$ will couple only weakly to the vortex, so we expect that $|\tau| \sim \gamma v_{1}$ everywhere.

Let us momentarily consider the limit $\alpha=\gamma=0$; the $\tau$ sector contributes nothing to the vacuum energy, and the $\phi$ and $\chi$ sectors decouple. Two independent strings are formed, one coming from the winding of $\phi$ and the other from the winding of $\chi$. Although these two vortices will each be cylindrically symmetric and stable, the two-vortex state has a trivial zero-mode describing the relative displacement of the two vortices. This zero mode becomes an instability when one allows $\alpha$ to be greater than zero while keeping $\gamma=0$, since now the vortex cores repel one another (with a short-range force). On the other hand, allowing $\gamma$ to be non zero while keeping $\alpha=0$ will also eliminate the zero mode, as the half-integer charge of $\tau$ confines the singly-charged vortices in pairs with $Q_{A}+Q_{B}=0 \bmod 2$. For $\alpha=0$ the ground state will be cylindrically symmetric, but 
taking $\alpha$ finite and increasing $\gamma$ from zero will clearly lead to an asymmetric ground state, since in that case the confining force is long-range but small. One can now argue (as in the first example of this section) that allowing both $\alpha$ and $\gamma$ to be greater than zero one will obtain a curve of marginal stability in the positive quadrant of the $\alpha, \gamma$ plane; the part of this quadrant between the axis $\gamma=0$ and the curve of marginal stability is the region of parameter space for which doubly-charged, cylindrically-symmetric vortices are unstable to formation of confined pairs of singly-charged vortices, i.e. binary strings.

Our initial restriction on the other scalar self-couplings is once again inessential. One can now add two more dimensions (corresponding to $\kappa_{1}$ and $\kappa_{2}$ ) to our parameter space, and the region of parameter space for which the binary string will be the ground state will be determined by some inequalities among the different contributions to the kinetic and potential energies. Since even a modest hierarchy among the vev's $v_{i}$ is sufficient to ensure that these inequalities pose little restraint on the parameter space, we see that for these models the binary string is technically natural and even generic, in the same sense that type I and type II vortices both occur in the phenomenology of superconductors.

\subsection{Goldstone modes and the binding force}

An interesting feature of the above models is the presence of a Goldstone boson in the broken phase. In both models we have omitted a scalar self-coupling which links the phase rotations of the different fields, and as a result there is an extra $U(1)$ symmetry which is not gauged and is spontaneously broken along with the gauged U(1)'s. This extra global symmetry will be preserved by renormalization if originally present (unless, e.g., there are fermions and the global symmetry is anomalous), and could arise as a result of discrete symmetries in a more fundamental theory, or through the absence of suitable renormalizable couplings in a unified model. In realistic models there are no massless scalars, and so either the symmetry-breaking coupling is present, or else perhaps the extra $\mathrm{U}(1)$ can be gauged. When the symmetry-breaking coupling is small enough the binary strings will exist by our earlier continuity argument.

Let us define what we mean by the binding force. Consider quasi-statically separating the components of the string transversely along their length. Although these strings cannot be divided into their constituent strings, there will be deformations that stretch the binary string into two string-like boundaries of a domain-wall-like structure. Thus, consider a one-parameter family of deformations of the binary vortex in the first model, where the field $\phi$ is constrained to vanish at two different points in the transverse plane at a distance 
$R$. Finding the minimum energy solution to the equations of motion with this constraint, we define the binding force as (-) the derivative of the energy with respect to the parameter $R$.

The fields forming the domain wall are massive in the true vacuum and the domain wall has a thickness $h$ of order $\frac{1}{m}$. The energy of the domain wall is then a linear function of its area when the area is large compared to the thickness. For binary strings this translates to a binding force that is constant when $R$ is large compared to the thickness of the domain wall. When the 'domain wall' is relatively thick $(h>>R)$ the energy grows less than linearly with the separation $R$, perhaps logarithmically. In fact, for small $R$ the fields in the region between the strings generally do not resemble a domain-wall. This small-separation case is quite typical of grand-unified models of binary strings, since the binding force and the short-distance repulsion may come from vastly different energy scales. Moreover, the nature of the binding force affects the action describing the motion of the binary string [5].

If the Goldstone mode is present, again the restriction to values of $R$ much larger than $h$ is insufficient. Nevertheless, by its nature the Goldstone mode is derivatively coupled and for large enough $R$ the contribution from the massive fields to the domain wall energy will dominate the effects of the Goldstone boson due to their linear increase with $R$. Because of the strong changes in the fields near the string cores, the Goldstone boson may couple strongly to the motion and internal excitation of the strings.

\section{Molecular binaries}

Finally we consider models in which the attractive force between the two vortices or strings is short-ranged, and the existence of binary strings is due to a minimum in the vortex-vortex potential. The energy required to separate two vortices is then finite, but for strings of cosmological length, separation is energetically impossible. Consider a $U(1) \times \tilde{U}(1)$ gauge theory with complex scalar fields $\phi_{1}, \phi_{2}$ and a neutral scalar $\sigma$, with charges $(1,0),(0,1)$ and $(0,0)$ respectively. The Lagrangian is,

$$
L=\frac{1}{2}\left|D \phi_{1}\right|^{2}-\frac{1}{4} F_{\mu \nu} F^{\mu \nu}+\frac{1}{2}\left|D \phi_{2}\right|^{2}-\frac{1}{4} G_{\mu \nu} G^{\mu \nu}+\frac{1}{2}|\partial \sigma|^{2}-V\left(\phi_{1}, \phi_{2}, \sigma\right),
$$

where $F$ and $G$ are the field strengths of $A$ and $B$ respectively, $D_{\mu} \phi_{1}=\left(\partial_{\mu}+i e A_{\mu}\right) \phi_{1}$, and $D_{\mu} \phi_{2}=\left(\partial_{\mu}+i e B_{\mu}\right) \phi_{2}$. The potential is

$$
V\left(\phi_{1}, \phi_{2}, \sigma\right)=\lambda_{1}\left(\left|\phi_{1}\right|^{2}-\mu_{1}^{2}\right)^{2}+\lambda_{2} \sigma^{4}+m^{2} \sigma^{2}-\lambda_{3}\left(\mu_{1}^{2}-\left|\phi_{1}\right|^{2}\right) \sigma^{2}+
$$




$$
\lambda_{4}\left(\left|\phi_{2}\right|^{2}-\mu_{2}^{2}\right)^{2}-\lambda_{5}\left(\mu_{2}^{2}-\left|\phi_{2}\right|^{2}\right)\left(\mu_{1}^{2}-\left|\phi_{1}\right|^{2}\right)+\lambda_{6}\left(\mu_{2}^{2}-\left|\phi_{2}\right|^{2}\right) \sigma^{2} .
$$

Note that the off-diagonal quartic couplings $\lambda_{5}$ and $\lambda_{6}$ have the 'wrong sign'; for a stable vacuum to exist this potential must be bounded from below. If the masses and coupling constants are all positive, a sufficiency condition for the positivity of the energy is easy to write: defining $V=V^{\prime}+m^{2} \sigma^{2}$ we see that the positivity of $V^{\prime}$ is sufficient to guarantee the positivity of $V$.

Now define $x_{1}=\mu_{1}^{2}-\phi_{1}^{2}, x_{2}=\sigma^{2}, x_{3}=\mu_{2}^{2}-\phi_{2}^{2}$, and in a matrix notation we can write $V \prime=X^{T} \Lambda X$; where $X=\left(x_{1} x_{2} x_{3}\right)$ and

$$
\Lambda=\left(\begin{array}{ccc}
\lambda_{1} & -\lambda_{3} / 2 & -\lambda_{5} / 2 \\
-\lambda_{3} / 2 & \lambda_{2} & \lambda_{6} / 2 \\
-\lambda_{5} / 2 & \lambda_{6} / 2 & \lambda_{4}
\end{array}\right)
$$

$V$ ' is positive definite if $\Lambda$ has no negative eigenvalues, which implies that the coefficients of the characteristic equation of $\Lambda$ must all be non-negative. This gives rise to some inequalities involving the couplings:

$$
\begin{gathered}
\operatorname{Tr} \Lambda=\lambda_{1}+\lambda_{2}+\lambda_{4}>=0, \\
\operatorname{det} \Lambda=4 \lambda_{1} \lambda_{2} \lambda_{4}+\lambda_{3} \lambda_{5} \lambda_{6}-\lambda_{1} \lambda_{6}^{2}-\lambda_{2} \lambda_{5}^{2}-\lambda_{3}^{2} \lambda_{4}>=0, \\
4\left[\lambda_{1} \lambda_{2}+\lambda_{2} \lambda_{4}+\lambda_{4} \lambda_{1}\right]-\lambda_{3}^{2}-\lambda_{5}^{2}-\lambda_{6}^{2}>=0 .
\end{gathered}
$$

When these conditions are satisfied the potential energy can be written as a sum of squares by diagonalizing $\Lambda$ so it is explicitly non-negative, furthermore, we have arranged that $V=0$ in the desired vacuum. We will assume that all the $\lambda$ 's are positive thus satisfying (3.4)at once. We shall come back to the other conditions on the $\lambda$ 's later, when the other requirements are known.

First let us note that with this Higgs potential, the $U(1) \times \bar{U}(1)$ symmetry is spontaneously broken; the vacuum expectation value of the symmetry breaking Higgs fields are $\left|\phi_{1, v e v}\right|=\mu_{1}$ and $\left|\phi_{2, v e v}\right|=\mu_{2}$; and the vev of the singlet field $\sigma$ is zero in the true vacuum. The manifold of vacua has nontrivial topology; $\pi_{1}(U(1) \times \tilde{U}(1))=Z \times \tilde{Z}$ implies the existence of two kinds of strings: those with a flux of $A$ and those with a flux of $B$. We shall call them type A and type B vortices, respectively. These can occur independently, so the binding which occurs is through short-range forces only. As we shall see, the vortex-vortex binding can occur between vortices of different types. 
Vortices of circulation one in either A or B alone are always stable. We are interested in the case of a pair of parallel A and B type strings with a vev of $\sigma$ in the core of the A type string. Let us review the conditions for which $\sigma$ would get a vev in the core of the A type string. The terms in the potential involving $\sigma$ are,

$$
V(\sigma)=\lambda_{2} \sigma^{4}+m^{2} \sigma^{2}-\lambda_{3}\left(\mu_{1}^{2}-\phi_{1}^{2}\right) \sigma^{2}
$$

At the core of A type string we can write a local potential for $\sigma$ with other fields replaced by their values at $r=0, \phi_{1}=0, \phi_{2}=\mu_{2}$ :

$$
V(\sigma)=\lambda_{2} \sigma^{4}+m^{2} \sigma^{2}-\lambda_{3}\left(\mu_{1}^{2}\right) \sigma^{2}
$$

There is a non trivial minimum of this potential at $\sigma_{v e v}^{2}=\frac{\lambda_{3} \mu_{1}^{2}-m^{2}}{2 \lambda_{2}}$, when $\lambda_{3} \mu_{1}^{2}>m^{2}$.

We can write a similar potential at the core of a string of type B; however, the sign of the term with $\lambda_{6}$ in the potential has been chosen so that the minimum of the 'core' potential is now at $\sigma_{v e v}=0$. The true configuration of the $\sigma$ field inside the A vortex is of course dictated by a balance between the gradient and potential contributions to the energy. However, when the condition

$$
\lambda_{3} \mu_{1}^{2}>m^{2}
$$

is fulfilled, a nonzero value of $\sigma_{v e v}$ is preferred at the core of the A type strings because of the gain in potential energy.

The conditions for obtaining a condensate of a 'spectator' scalar field were examined in [2]; let us briefly recapitulate. We begin with a static, cylindrically-symmetric vortex solution (Nielsen-Olesen vortex) for $\phi_{1}$ and the gauge field $A_{\mu}$, neglecting $\phi_{2}$ for now. We wish to see if $\sigma=0$ is indeed the vacuum state; we examine small oscillations of $\sigma$ around the background $\sigma=0, \sigma=\sigma_{0}(r) e^{i \omega t}$. The linearized equation of motion of $\sigma$ is,

$$
-\frac{\partial^{2} \sigma_{0}(r)}{\partial r^{2}}-\frac{1}{r} \frac{\partial \sigma_{0}(r)}{\partial r}-P \sigma_{0}(r)=\omega^{2} \sigma_{0}(r)
$$

When there are solutions of (3.10) with $\omega^{2}<0, \sigma=0$ is an unstable solution of the classical equations [2]. When $m^{2}=0$, the potential energy of this 2d Schroedinger equation, $P \equiv \lambda_{3}\left(\mu_{1}^{2}-\phi_{1}^{2}\right)$ is everywhere negative when the background is the Nielsen-Olesen vortex solution $\phi_{1}(N . O)$. The argument in [2] then proceeds that, since a negative definite potential in two dimensions always has a bound state, $\omega^{2}$ has a negative value for $m^{2}=0$; 
by continuity, then, in the neighborhood of $m^{2}=0, \sigma=0$ is an unstable solution which relaxes to a non zero vev for $\sigma$. Indeed, $\sigma$ develops a nonzero vev whenever $m^{2}<\mu^{2}$, where $-\mu^{2}$ is the most negative eigenvalue for $\omega^{2}$ in equation (3.6) with $m=0$.

Although we cannot express $\mu$ directly in terms of the parameters of the theory, it can be easily approximated by doing a simple variational calculation, or even more simply, estimated by making an ansatz for the zero mode, parametrizing the shape of the vortex by elementary functions. Choosing the latter method (taking the ansatz for $\sigma_{0}$ to be given by the difference of two exponentials) we get

$$
\omega^{2} \sim 2.4 k^{2}-0.6 \lambda_{3} \mu_{1}^{2} .
$$

where $k \equiv \sqrt{2 \lambda_{1}} \mu_{1}$. The first term on the $R H S$ is from the kinetic energy and the second is from the potential energy of the $\sigma$ condensate. There are two things to note: (a) This value of $\omega^{2}$ underestimates the actual value of $\mu^{2}$. However, we can also obtain an upper bound; for a vortex with $\left|\phi_{1}(r)\right|$ that monotonically increases from $\phi_{1}=0$ at $r=0$ to $\phi_{1}=\mu_{1}$ at $r=\infty$, we must have $\mu^{2} \leq \lambda_{3} \mu_{1}^{2}$. (b) The value of $\mu^{2}$ is an increasing function of the range of the potential, when the strength of the potential is held constant. This is because the potential energy term $P(r)$ increases in magnitude point-wise as the range of the potential is increased. In our case the range of the potential is $1 / k$, so $\mu^{2}$ is an increasing function of $\mathrm{k}$.

We want to show that there is a range of parameters for which (i) $\sigma$ develops a vev in the core of the A type strings; and (ii) the asymptotic forms of the $\phi_{1}$ and $\sigma$ fields is $\left|\phi_{1}\right|(r) \sim \mu_{1}-\phi_{1} e^{-k r}$ and $|\sigma|(r) \sim \sigma_{0} e^{-m r}$. (i) will be true if the condition $m^{2} \leq \mu^{2}$ is satisfied. Since $\mu^{2} \leq \lambda_{3} \mu_{1}^{2}$ we can define a vortex parameter $f_{\phi_{1}}$ (depending on the vortex field background $\phi_{1}$ ) by

$$
\left|\mu^{2}\right|_{\phi_{1}}=f_{\phi_{1}} \mu_{1}^{2} \lambda_{3},
$$

with $0<f_{\phi_{1}}<=1$.

Condition (ii) for $|\sigma|(r)$ simply follows from the linearized classical equations in the asymptotic region. Similarly we obtain the correct asymptotic behavior for $\phi_{1}$ if $m^{2}>$ $8 \lambda_{1} \mu_{1}^{2}$ and $e^{2}>2 \lambda_{1}^{2}$. For consistency with (i) we then must have,

$$
f_{\phi_{1}\left(\lambda_{1}\right)} \lambda_{3}>8 \lambda_{1} .
$$

For given values of $\mu_{1}^{2}$ and $\lambda_{3}$ there exist a range of values for $\lambda_{1}$ for which this consistency condition is satisfied. This is quite natural since the width of the Nielsen-Olesen vortices 
increases as $\lambda_{1} \rightarrow 0$; in that limit, $\mu^{2}$ tends to its maximum value: $\mu^{2} \rightarrow \lambda_{3} \mu_{1}^{2}$. Thus, given any $\lambda_{3}>0$, there is a range of values of $\lambda_{1}$ extending to zero satisfying $f_{\phi(N . O)} \lambda_{3}>8 \lambda_{1}$.

Having established the asymptotic behavior of the $\phi_{1}$ and $\sigma$ fields when $f_{\phi_{1}} \lambda_{3}>\lambda_{1}$ we now examine the behavior of a B-type vortex in the background of an A-type vortex, which as we see has a $\sigma$ condensate in the core. The scale that determines the width of the B type vortex is the mass of the $\phi_{2}$ field in the true vacuum, width $(\mathrm{B}) \sim \frac{1}{\sqrt{\lambda_{2} \mu_{2}^{2}}}$. We assume that the $\left(\phi_{1}, \sigma\right)$ and the $\phi_{2}$ sectors have a large difference in mass scales, $\mu_{2}^{2}>>\mu_{1}^{2} \sim m^{2}$. This would be natural for $\lambda_{5}=\lambda_{6}=0$, when the two sectors are decoupled. In a GUT version this hierarchy would result from symmetry breaking at different scales; in the present case, it does not need to be very large. In this simplified model we assume $\lambda_{5}, \lambda_{6}$ to be relatively small: $\lambda_{2}, \lambda_{3}, \lambda_{4}>>\lambda_{5}, \lambda_{6}$.

The large mass scale of the $\mathrm{B}$ type vortex ensures that the B type strings are very thin compared to the A type ones. We will then approximate the 'shape' of the B vortex effectively by a delta function, with the field $\phi_{2}$ dropping abruptly to zero from its true vev $\mu_{2}$ at the core of the vortex, for the purpose of considering the interaction of the vortices. If we bring such a (thin) B-type string near an A-type string, while keeping them parallel, the structure of the vortices will not be changed appreciably by their mutual interaction. Indeed the interaction is purely through quartic Higgs couplings of $\lambda_{5}$ and $\lambda_{6}$, which can be treated as a perturbation.

We can now calculate the interaction energy per unit length of a pair of parallel A and B type strings, $V_{\text {int }}=\mu^{\prime 2}\left[\lambda_{6} \sigma^{2}\left(r^{\prime}\right)-\lambda_{5}\left(\mu_{1}^{2}-\phi_{1}^{2}\left(r^{\prime}\right)\right)\right]$, where $\mu^{\prime} \sim \mu_{2}^{2}$ is determined by integrating the interaction energy over the cross section of the thin B-type vortex, and $r^{\prime}$ is the position of the B-string relative to the core of the A-string.

When the B string is far away from the core of the A string we can use the asymptotic forms of the $\phi_{1}$ and $\sigma$ fields:

$$
\begin{gathered}
\left|\phi_{1}(r)\right| \simeq \mu_{1}-\phi_{1} e^{\sqrt{-8 \lambda_{1}} \mu_{1}(r)} ; \\
|\sigma(r)| \simeq \sigma e^{-m r} ; \\
V_{\text {int }}=\mu_{2}\left[\lambda_{6} \sigma^{2} e^{-2 m r}-2 \lambda_{5} \mu_{1} \phi e^{-\sqrt{8} \lambda_{1} \mu_{1}(r)}\right]+\text { h.o. }
\end{gathered}
$$

At $r \rightarrow \infty, \frac{\partial V_{i n t}}{\partial r}$ is positive $\left(m^{2}>2 \lambda_{1} \mu_{1}^{2}\right)$, giving an attractive force between the vortices. This force decreases to zero and changes sign at the equilibrium point $r=r_{e q}$ which is given by $2 \lambda_{6} \sigma^{2} m e^{-2 \omega r_{e q}}-2 \lambda_{5} \mu_{1} \phi_{1} e^{-\sqrt{\lambda_{1} \mu_{1}\left(r_{e q}\right)}}+$ h.o. $=0$. 
The vortex-vortex interaction leads to a equilibrium separation of the strings, $r_{e q}$. By choosing an appropriate ratio of the constants $\lambda_{5}$ and $\lambda_{6}, r_{e q}$ can be made to fall within the range of validity of our approximations. In the present case, $\lambda_{6}$ must be much larger than $\lambda_{5}$. (That is, we have balanced a somewhat-longer range attraction with a stronger, but shorter ranged repulsion.) This will obviously lead to a binding energy per unit length between the two strings that is several orders of magnitude smaller than the mass per unit length of the binary string, since the attractive force is proportional to $\lambda_{5}$. To get a larger binding energy one could increase $\lambda_{5}$ (and then $\lambda_{6}$ as well), in which case one must replace the asymptotic forms of the $\phi_{1}$ and $\sigma$ fields by the exact classical solution, since $r_{e q}$ moves into the core of the A-string. There is a priori no reason that the qualitative features would change drastically for more strongly bound strings; there will be some range of couplings for which the binary-string ground state is obtained, with the limit on the strong-binding side being set by the fact that as the couplings get stronger and the strings are more closely bound, it becomes energetically favorable to deform the A-string and obtain a cylindrically-symmetric configuration. In any case we have an existence proof of vortex-vortex binding at finite separation in this model, and presumably others of the same class can be found.

(Returning to the constraints on our scalar potential, the nontrivial conditions (3.5), (3.6) and (3.12) in the above model are met for a wide range of (positive) parameters $\lambda$. For instance when $\lambda_{5}$ and $\lambda_{6}$ are sufficiently small the conditions essentially reduce to

$$
\begin{gathered}
4 \lambda_{1} \lambda_{2} \lambda_{4}-\lambda_{3}^{2} \lambda_{4}>\delta, \\
4\left[\lambda_{1} \lambda_{2}+\lambda_{2} \lambda_{4}+\lambda_{4} \lambda_{1}\right]-\lambda_{3}^{2}>\delta, \\
f_{\phi} \lambda_{3}>8 \lambda_{1},
\end{gathered}
$$

where $\delta$ is a small positive parameter. If $\lambda_{1}=\epsilon f_{\phi} \lambda_{3}$, with $\epsilon<1$, then conditions (3.5), (3.6) and (3.12) are easily satisfied by any partial ordering $\lambda_{2}, \lambda_{4}>\lambda_{3}$ satisfying $4 \lambda_{2}>$ $\lambda_{3} / \epsilon f_{\phi}$.)

To compare this with the previously-constructed models, the most significant difference is the finite binding-energy per unit length, which is much smaller than the string tension. The individual strings in this binary are independently stable; if they were initially separate, they would be very unlikely to combine because the interaction energy between two intersecting segments of string could not compete with the kinetic energy of the rest of the string under normal circumstances. On the other hand, if such a binary string were formed it would be very difficult to dissociate, since a macroscopic string would have a very large binding energy, and it would be necessary to transfer sufficient energy to the whole string, rather than an individual segment. 


\section{GUT considerations}

Binary strings of the 'confining' type will occur in grand-unified theories provided two conditions are satisfied: the sequence of symmetry-breaking phase transitions gives rise to cosmic strings and broken discrete symmetries in the necessary order, and the coupling constants are such that multiple-winding strings are unstable. The first of these conditions is the more stringent one. The question of whether the pattern of symmetry breaking is appropriate depends on the representations of the symmetry-breaking order parameters (e.g.Higgs fields) and the scales at which the symmetries are broken. (Note that although we will be describing the symmetry breaking in terms of elementary scalar fields, there is no reason why other symmetry-breaking mechanisms, such as fermion condensates or Wilson lines, could not play a role).

The first type of confining binary, in which two similar strings are bound together by a (vestigial) domain wall, is the most generic, since it could occur in any grand-unified model in which strings are first formed and at a later phase transition bound domain walls. It may be surprising that a stable remnant persists in this case; however, we observe that the orientation of the domain wall is not gauge-invariant, but changes direction under the $Z_{2}$ discrete gauge symmetry spontaneously broken by the lighter scalar field, so the string can assume either orientation as the boundary of the domain wall. (In terms of the holonomy of the gauge field, the same group element, $(-1)$ in the representation of the charge-1 field, is obtained in passing around the string in either direction, and likewise passing in either direction through the domain wall.) In the next section, we remark on how this circumstance allows $(n=2)$ strings, whether binary or not, to survive the phase transition in which the domain walls form.

This type of confining behavior can occur in a well-known model of $\mathrm{SO}(10)$ grandunification described by [7] in their analysis of string-bounded domain walls. In that symmetry breaking sequence,

$$
\operatorname{Spin}(10) \stackrel{\mathbf{5 4}}{\longrightarrow} \operatorname{Spin}(6) \otimes S p i n(4) \stackrel{\mathbf{1 2 6}}{\longrightarrow} S U(3) \otimes S U(2) \otimes U(1) \stackrel{\mathbf{1 0}}{\longrightarrow} S U(3) \otimes U(1)
$$

cosmic strings are formed (of the 'Alice string' variety [8] [9]) at the first phase transition; the 126 vev is not $\mathcal{C}$-invariant, and so at the second phase transition the strings bound domain walls. The surviving strings will be of the binary or the $n=2$ type, depending on the relative sizes of the scalar and gauge couplings, as previously discussed. Other examples may be similarly constructed. 
Incidently, this same model (in a different region of parameter space) might exhibit the 'molecular' variety of binary string. At the phase transition in which the domain walls are formed, there are independently formed [7] stable cosmic strings (corresponding to the discrete gauge symmetry $\left.(-1)^{F_{10}} \in \operatorname{Spin}(10)\right)$. They might be expected to be comparable in abundance to the remnant $n=2$ strings remaining after the domain walls have disappeared. One could then extend the scalar sector of this model so that the situation of section 4 is realized, that is, a weak attraction at long distances between the strings, and a short-distance repulsion, due to a scalar condensate in the core. In view of the somewhat dubious cosmology of this type of defect, we will not develop such a model in detail.

If we consider grand-unification based on the $E_{6}$ gauge group, there are more possibilities for the unbroken discrete gauge symmetries and hence a variety of string solutions. So, for instance, if at the highest scale we break $E_{6}$ to $S U(5) \otimes U(1) \otimes U(1)$ via one or more adjoints (78's), we can obtain independent cosmic strings at subsequent stages of symmetry breaking, either in combination with or independent of $S U(5)$ breaking. These could furnish examples of any of the varieties of binary string we have described.

We will present one concrete example along a somewhat different line. Consider an $E_{6}$ GUT model, with a symmetry-breaking sequence

$$
E_{6} \stackrel{\mathbf{2 7}}{\longrightarrow} S O(10) \otimes Z_{4} \stackrel{\mathbf{3 5 1}^{\prime}}{\longrightarrow} S U(5) \times Z_{2} \otimes Z_{2} \stackrel{\mathbf{2 7}}{\longrightarrow} S U(5) \otimes Z_{2} \rightarrow \ldots
$$

(we leave the subsequent symmetry breaking to the reader's imagination.) The representations of the scalar vev's are, respectively, a $\mathbf{2 7} \phi$, a $\mathbf{3 5 1 ^ { \prime }} \chi$, and another $\mathbf{2 7} \tau$. (Note that the $\mathbf{3 5 1}$ ' is in the symmetric product of two $\overline{\mathbf{2 7}}$ 's, and the direction of the vev is chosen to lie in the 126 direction in its $S O(10)$ decomposition, so the $Z_{2}$ string is similar to those in $S O(10)$ GUT models). Our nicknames for the scalars is meant to suggest the similarity to the second model of section 2; indeed, the only difference is that in this case the discrete symmetry group has a different breaking pattern, $Z_{4} \otimes U(1) \rightarrow Z_{2} \otimes Z_{2} \rightarrow Z_{2}$. Because of the $Z_{4}$ breaking the composite strings may form at the second phase transition, as well as at the third.

Finally, we once again emphasize that the point of this exercise is not to find compelling grand-unified models with binary strings, although the first example would do for that, but rather to show that in non-minimal unification the conditions for the existence of composite strings are easily satisfied. 


\section{Formation and Evolution of Binary Cosmic Strings}

The formation of binary cosmic strings begins by the Kibble mechanism [10]: the Higgs scalar develops an expectation value below some critical temperature $T_{c}$, which is disordered on scales larger than some correlation length $\xi_{\phi}$ determined by the kinetics of the phase transition. As the scalar field relaxes, defects are formed at the boundaries of domains with different orientations of the condensate. At lower temperatures, the scalar field fluctuations are suppressed and the network of defects begins to evolve.

The details of binary string formation depend on the mechanism responsible for the internal structure and the evolution subsequent to the initial formation of the strings. In the case of confined binary strings domain walls are formed at a later phase transitions when one of the discrete gauge symmetries is broken. Since the strings arising from the earlier symmetry breaking are charged under the discrete symmetry, they will become boundaries of domain walls. As the network of domain walls forms in the presence of the strings the relative orientations of different components of the boundary of a given wall is determined by chance. Then, as the network evolves, some components of the boundary will meet as the domain wall shrinks; those that have opposite orientations will annihilate, while those segments of string that have the same orientation will form linear defects with winding number $n=2$. These will be binary strings, when the couplings are such that the cores are repulsive; otherwise they will form metastable, cylindrically-symmetric $n=2$ strings. (In both cases the strings are unstable to decay by $Z_{2}$ monopole-pair production [11], through a greatly suppressed tunneling process; because of the separation of the cores the rate will be even more heavily suppressed in the case of binary strings.)

The situation is similar with the other confining model, in which two different types of string are bound together at the discrete-symmetry-breaking phase transition. Here, however, the domain-wall can end with either orientation, on either of the types of string. Generally we may assume one of these strings is much lighter and more abundant than the heavier ones initially formed. It is then reasonable to expect that while many of the lighter strings may annihilate, nearly all of the heavier strings should be paired up with the more abundant lighter strings. (Note that in this case if the couplings do not make the cores mutually repulsive, the final state will have strings with a gauge-field flux being a composite of that comprising the initial strings.)

The circumstances under which the 'van der Waals' or 'molecular' strings may be formed cosmologically are quite unclear. There may be circumstances under which preexisting strings from an earlier phase transition could serve as nucleation sites for the 
subsequent transition or otherwise influence the ordering of the scalar field condensate, in such a fashion that the binary strings are formed together. Otherwise, since the separate strings are stable and the interaction in this case is short-ranged, there is vanishing probability of forming binary strings when the component strings are formed separately.

After formation the evolution of a network of binary cosmic strings is essentially the same as for ordinary cosmic strings, since at large distances the string mechanics is dominated by the string tension (which is nearly the sum of the tension for each component). One difference is that the initial network of strings formed by the collapse of the domain walls may be somewhat different from the usual string network, but differences of this type will presumably be washed out by the subsequent evolution of the string network. Another effect (albeit a small one) which should be noted is that the effective string tension and mass on cosmological scales [12] will be affected by the internal degrees of freedom (twisting modes).

It is more interesting consider the coupling of the internal modes of the binary cosmic string to the various epiphenomena of cosmic string solutions: superconductivity, fermion zero modes, the kinks and cusps of classical string evolution. Some of these questions will be addressed in the companion paper [5].

\section{Acknowledgments}

This work was supported by DOE contract \#DE-FG02-91ER40767. 


\section{References}

[1] H.B. Nielsen and P. Olesen, Nucl.Phys. B61 (1973) 45.

[2] E. Witten, Nucl.Phys. B249 (1985) 557.

[3] Ya.B. Zel'dovich, Mon.Not.Roy.Astron.Soc. 192(1980) 663

[4] A. Vilenkin, Phys.Rev.Lett. 46 ( 1981) 1169,1496(E); Phys. Rev. D24 (1981) 2082.

[5] I. Dasgupta and R. Rohm, "Binary String Dynamics," in preparation.

[6] E.B. Bogomol'nyi, Sov.J.Nucl.Phys. 24 (1976) 449.

[7] T.W.B. Kibble, G. Lazarides and Q. Shafi, Phys.Rev. D26 (1983) 435.

[8] A.S. Schwarz, Nucl.Phys. B208 (1982) 141; A.S. Schwarz and Yu.S. Tyupkin, Nucl.Phys. B209 (1982) 427.

[9] M.G. Alford, K. Benson, S. Coleman, J. March-Russell and F. Wilczek, Phys.Rev.Lett. 64 (1990) 1632, (E)65 (1990) 668; J. Preskill and L. Krauss, Nucl.Phys. B341 (1990) 50.

[10] T.W.B. Kibble, J.Phys. A9 (1976) 1387

[11] A. Vilenkin, Nucl.Phys.B196 (1982) 240

[12] A. Vilenkin, Phys.Rev. D41 (1990) 3038; B. Carter, Phys.Rev. D41 (1990) 3869 